\title{
Annexin A1 Is a Potential Prognostic Marker for, and Enhances the Metastasis of, Cholangiocarcinoma
}

\author{
Kwuntida U Kotepui ${ }^{1,2,3}$, Sumalee Obchoei ${ }^{1,2,4}$, Kulthida Vaeteewoottacharn ${ }^{1,2}$, \\ Seiji Okada ${ }^{5}$, Sopit Wongkham ${ }^{1,2}$, Kanlayanee Sawanyawisuth ${ }^{1,2 *}$
}

\begin{abstract}
Objective: Annexin A1 (ANXA1) is a calcium-dependent phospholipid-binding protein which contributes to proliferation, cancer progression and metastasis. Overexpression of ANXA1 is closely associated with metastasis in numerous types of cancer. Cholangiocarcinoma (CCA) is a bile-duct cancer which has high rates of metastasis. Previously, we demonstrated up-regulation of ANXA1 in a highly metastatic CCA cell line (KKU-213AL5). Here, we investigated the functions of ANXA1 in the progression of CCA cell lines and evaluated its clinical impacts in human CCA tissues. Methods: Effects of ANXA1 on metastatic potential of CCA cell lines were evaluated using cell-proliferation, clonogenic, migration and invasion assays. The expression of ANXA1 in 44 intrahepatic human CCA tissues was investigated using immunohistochemistry (IHC). The association of ANXA1 with clinicopathological features of CCA patients was analyzed. Results: Silencing of ANXA1 expression using siRNA significantly decreased cell proliferation, colony formation, cell migration and invasion in the KKU-213AL5 cell line. IHC results showed low expression of ANXA1 in normal bile ducts in the non-tumor area. In contrast, high expression of ANXA1 in human CCA tissues was associated with advanced tumor stage, tumor size and presence of lymph-node metastasis. Conclusion: These findings strongly imply that ANXA1 contributes to the progression of CCA. ANXA1 can serve as a potential prognostic marker for CCA. Ablation of ANXA1 action may be an alternative strategy to prevent metastasis of CCA.
\end{abstract}

Keywords: Annexin A1- ANXA1- cholangiocarcinoma- metastasis

Asian Pac J Cancer Prev, 23 (2), 715-721

\section{Introduction}

Cholangiocarcinoma (CCA) is a bile duct cancer which is difficult to diagnose at an early stage. Its high invasiveness and frequent metastasis contribute to the devastat $\neg$ ing prognosis and notoriously high mortality rate of CCA (Zeng and Tao, 2015). Complete resection is the only option for a cure. However, only a very small proportion of CCA patients can receive such surgery (Luvira et al., 2016). Metastasis has already occurred in about $80 \%$ of CCA patients at diagnosis. These patients usually receive ineffective chemotherapy or palliative treatments (Butthongkomvong et al., 2013). Currently, there is little understanding of the molecular processes involved in metastasis in CCA that might give clues leading to a more effective treatment of this highly metastatic cancer.

Our previous study revealed high expression of annexin A1 (ANXA1) in a highly metastatic CCA cell line
(KKU-213AL5) relative to the parental KKU-213A cell line (Uthaisar et al., 2016). ANXA1 is a member of the annexin family which consists of calcium ion-dependent phospholipid-binding proteins (Caron et al., 2013). It plays a role in a variety of cellular biological activities including cell proliferation, cell death and carcinogenesis (Lim and Pervaiz, 2007). Increasing ANXA1 expression was observed during cholangiocarcinogenesis in a hamster model and was also found in human CCA cases (Wang et al., 2006a; Hongsrichan et al., 2013). Depletion of ANXA1 expression inhibited TGF- $\beta$ and MMPs but induced NF- $\kappa$ B expression in a CCA cell line (Hongsrichan et al., 2013). However, the function of ANXA1 in metastasis of CCA has not been clarified. Therefore, we aimed to investigate the cell proliferation and cell invasiveness in the highly metastatic KKU-213AL5 cell line following ANXA1 knockdown. Additionally, the clinical significance of ANXA1 was evaluated in human CCA tissues.

${ }^{1}$ Department of Biochemistry, Faculty of Medicine, Khon Kaen University, Khon Kaen 40002, Thailand. ${ }^{2}$ Center for Translational Medicine, Faculty of Medicine, Khon Kaen University, Khon Kaen 40002, Thailand. ${ }^{3}$ Medical Technology Program, School of Allied Health Sciences, Walailak University, Nakhon Si Thammarat 80161, Thailand. ${ }^{4}$ Department of Biochemistry, Faculty of Science, Prince of Songkla University, Songkhla 90110, Thailand. ${ }^{5}$ Division of Hematopoiesis, Joint Research Center for Human Retrovirus Infection \& Graduate School of Medical Sciences, Kumamoto University, 860-0811, Japan. *For Correspondence: kanlayanee@kkumail.com 


\section{Materials and Methods}

Human CCA tissues, CCA cell lines and metastases of these cell lines in mouse lungs

The KKU-213A CCA cell line, its highly metastatic derivative KKU-213AL5, and metastasized lung tissues from mice injected intravenously with KKU-213A and KKU-213AL5 cells were obtained as described previously (Uthaisar et al., 2016). The procedure was approved by the Institutional Animal Care and Use Committee of Kumamoto University . Paraffin-embedded human CCA tissues from histologically confirmed mass-forming type of CCA $(n=44)$ were obtained from the specimen bank of the Cholangiocarcinoma Research Institute, Khon Kaen University, Thailand. The protocol was reviewed and approved by The Khon Kaen University Ethics Committee for Human Research (HE591063) based on the Declaration of Helsinki and ICH-Good Clinical Practice Guidelines.

\section{Transient knockdown of ANXA1 using siRNA}

ANXA1 suppression was performed using siRNA; sense sequence: 5' GGACUUUGG UGUGAAUGAAtt 3' and antisense sequence: 5'UUCAUUCACACCAAAGUCCtc 3'. KKU-213AL5 cells ( 2 x $10^{5}$ cells $)$ were plated into a 6-well culture plate and transfected with 50 pmole $/ \mathrm{mL}$ si-ANXA1 or scramble control. Lipofectamine ${ }^{\mathrm{TM}} 2000$ (Invitrogen, CA, USA) was used as a transfection reagent. Cells were incubated at $37^{\circ} \mathrm{C}$ in a $\mathrm{CO}_{2}$ incubator for $24 \mathrm{~h}$ after transfection and used for further analysis.

\section{Cell proliferation assay}

KKU-213AL5 cells $\left(1 \times 10^{3}\right.$ cells/well $)$ were seeded into a 96-well plate and treated with scramble or siANXA1 silencers. Cell proliferation was determined on days 1-3 after transfection using an MTT-based method according to the manufacturer's recommendation (Invitrogen, CA, USA). Briefly, $10 \mu \mathrm{L}$ of MTT reagent (0.5 $\mathrm{mg} / \mathrm{mL}$ final concentration) was added to each well. After incubation for $4 \mathrm{~h}, 100 \mu \mathrm{L}$ of $0.01 \mathrm{~N} \mathrm{HCl}$ in isopropanol was added to dissolve the crystals and absorbance was measured at $540 \mathrm{~nm}$.

\section{Clonogenic assay}

Survival and proliferative activities were determined using a clonogenic assay (Franken et al., 2006). Briefly, 200 cells in Ham's F-12 with 10\% FBS were seeded in a 6-well plate and incubated in a $5 \% \mathrm{CO}_{2}$ incubator at $37^{\circ} \mathrm{C}$ for 7 days. Colonies (at least 50 cells) were fixed in $4 \% \mathrm{v} / \mathrm{v}$ paraformaldehyde, stained with $0.5 \% \mathrm{w} / \mathrm{v}$ crystal violet and counted.

\section{In vitro cell-migration and invasion assays}

The migration and invasion assays were performed using a Boyden chamber assay as described previously (Uthaisar et al., 2016). Briefly, Transwell ${ }^{\circledR}$ plates with 8 $\mu \mathrm{m}$ pore polycarbonate-membrane inserts were coated with Matrigel (BD Biosciences) overnight. The CCA cells $\left(3 \times 10^{4}\right)$ were seeded onto the upper chamber of the Transwell ${ }^{\circledR}$ unit. Ham's F-12 medium with $10 \%$ FBS was used as a chemoattractant in the bottom chamber. The cells were allowed to invade at $37^{\circ} \mathrm{C}$ and $5 \% \mathrm{CO}_{2}$ for $7 \mathrm{~h}$. Cells in the upper chamber were gently wiped off with a cotton bud. The membranes containing the migrating/ invading cells were fixed in $4 \% \mathrm{v} / \mathrm{v}$ paraformaldehyde, stained with $0.4 \% \mathrm{w} / \mathrm{v}$ sulphorhodamine $\mathrm{B}$ and counted by averaging 9 low-power fields.

\section{Real-time PCR}

Total RNA was isolated from cells with Trizol ${ }^{\circledR}$ reagent (Invitrogen, Carlsbad, CA) and $2 \mu \mathrm{g}$ of RNA was reverse transcribed into cDNA using High-Capacity cDNA Reverse Transcription kits (Applied Biosystems, Foster City, CA). Real-time PCR was performed in the LightCycler ${ }^{\circledR} 480$ system (Roche Diagnostics, Germany). The reaction mixture $(20 \mu \mathrm{L})$ contained cDNA template, Light Cycler $^{\circledR} 480$ SYBR Green I Master reagent and the following primers: ANXA1 (forward: 5'AACGCTTTGCTTTCTCTTG 3', reverse: 5'CTTCTGGTGGTAAGGATGG 3') or $\beta 2$ microglobulin (B2M) (forward: 5'AAGATG AGTATGCCTGCCG 3', reverse: 5' CGGCATCTTCAAACCTCC 3'). The PCR protocol consisted of an initial denaturation step of $95^{\circ} \mathrm{C}$ for $5 \mathrm{~min}$, followed by 50 amplification cycles with 10 $\mathrm{sec}$ at $95^{\circ} \mathrm{C}, 10 \mathrm{sec}$ at the annealing temperature of $60^{\circ} \mathrm{C}$ and $3 \mathrm{sec}$ at $72^{\circ} \mathrm{C}$. The expression levels of ANXA1 gene were normalized with the B2M gene as internal control. The values were expressed as $\left(2^{-\Delta \mathrm{cp}}\right)$, where $\Delta \mathrm{cp}=$ [cp target gene-cp internal control and for fold change as $\left(2^{-\Delta \Delta \mathrm{cp}}\right)$, where $\Delta \Delta \mathrm{cp}=$ [cp target gene-cp internal control (tested cell) $] /[\mathrm{cp}$ target gene-cp internal control (control cells)].

\section{Cell lysis, SDS-PAGE and western blot analysis}

Cells were lysed with lysis buffer containing protease inhibitor cocktails. The protein concentration of cell lysate was determined using the Bradford assay (Bradford, 1976). Twenty-five micrograms of protein was separated on $10 \%$ SDS-PAGE and then transferred to a PVDF membrane using Bolt \& Mahoney transfer buffer according to the method described (Laemmli, 1970). The PVDF membrane was incubated with 1:1000 rabbit anti-ANXA1 polyclonal antibody (Abcam, MA, USA) or $1: 10,000$ anti- $\beta$-actin antibody (Sigma-Aldrich, MO, USA) followed by 1:20,000 HRP-linked secondary antibody (Zymed Laboratories, CA, USA). The immunoreactive bands were detected using the Chemiluminescence ECL Prime Western Blotting Detection System (GE Healthcare, IL, USA). The intensities of bands were analyzed using Image JTM analysis software.

\section{Immunohistochemistry (IHC)}

ANXA1 protein in the paraffin-embedded tissues was detected using a standard protocol. In brief, the sections were incubated with a 1:100 dilution of anti-ANXA1 antibody (Santa Cruz Biotechnology, CA, USA) at $4^{\circ} \mathrm{C}$ overnight and further incubated with the EnVision+system-HRP labeled polymer (Dako, Denmark). For cytokeratin 19 (CK19) (Sigma-Aldrich, MO, USA), the protocol was as described previously (Uthaisar et al., 2016). The frequency of ANXA1-positive cells was semi-quantitatively scored as $0 \%=$ negative; $1-25 \%$ positive cells $=+1 ; 26-50 \%=+2$; and $>50 \%=$ 
+3 . The intensity of positive staining was scored as weak $=1$, moderate $=2$ and strong $=3$. ANXA 1 expression was evaluated using an IHC index (intensity $\mathrm{x}$ frequency, range $0-9)$ and categorized as low $(\leq 6)$ or high $(>6)$.

\section{Statistical analysis}

Statistical analyses were performed using Sigma Stat version 3.5 software (Systat Software Inc., UK) and GraphPad Prism software (CA, USA). Student's t-test was used for comparisons between two groups. A $\mathrm{P}$-value $<0.05$ was considered statistically significant. ANXA1 expression was evaluated for association with clinicopathological findings using the Chi2-test $(* \mathrm{P}<0.05, * * \mathrm{P}<0.01$ and $* * * \mathrm{P}<0.001)$.

\section{Results}

High expression of ANXA1 in the highly metastatic KKU$213 A L 5$ cell line and in lung metastases due to this cell line

ANXA1 expression levels in KKU-213A and KKU-213AL5 CCA cell lines were compared at the mRNA level using real-time PCR and at the protein level using western blot. As shown in Figure 1A-B, ANXA1 expression was elevated in the highly metastatic KKU-213AL5 compared with the parental KKU-213A cell line. The greater metastatic potential of the KKU-213AL5 cells relative to the parental KKU-213A cells described previously. KKU-213AL5-injected mice developed $>10$-fold more lung metastatic foci than did those injected with KKU-213 cells. Mice injected with KKU-213AL5 also had shorter survival times (median of 21 days) compared to 36 days for those injected with KKU-213A cells (Uthaisar et al., 2016). We further verified the expression of ANXA1 in lung metastases from mice injected with KKU-213A or KKU-213AL5 using IHC staining (Figure 1C). CK19 staining confirmed that the lung metastases were indeed due to these biliary epithelial cancer cells. ANXA1 was expressed at a higher level in lung metastases of KKU-213AL5 relative to those of the KKU-213A cell line. The percentage of positive staining for ANXA1 in lung metastases from mice injected with KKU-213AL5 cells (100\%, 6/6, IHC index: 6.0 $\pm 2.7)$ was significantly higher than for KKU-213A (33\%, 2/6, IHC index: $2.2 \pm 3.7)$, as presented in Figure 1D $(\mathrm{P}<$ $0.05)$. These results imply that ANXA1 may play roles in metastasis of CCA cells.

Knockdown of ANXA1 caused reduction in cell proliferation, colony formation, cell migration and invasion of $K K U-213 A L 5$ cells

To examine the functions of ANXA1 in CCA progression, we knocked down the ANXA1 expression using RNA interference in the KKU-213AL5 cell line. The specific silencer of ANXA1 (si-ANXA1) effectively decreased the expression of ANXA1 protein to $45 \%$ of that
A

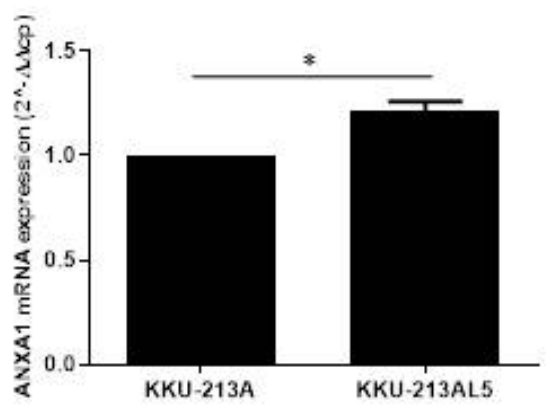

C

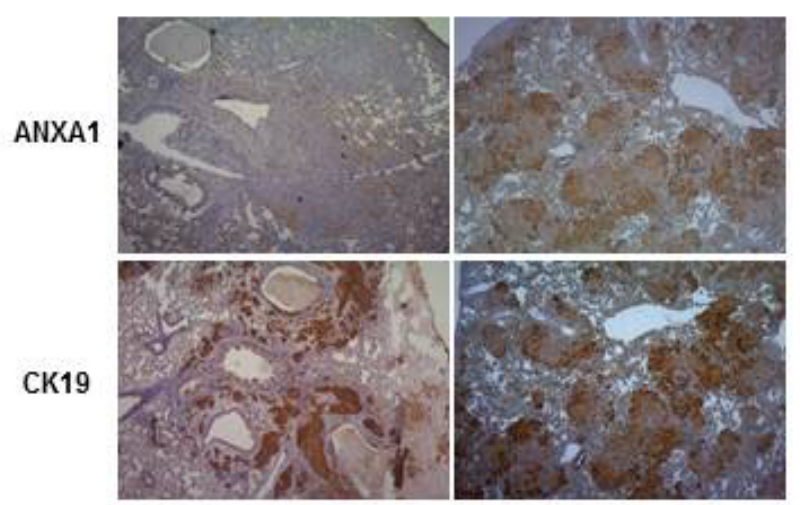

B

KKU-213A KKU-213AL5

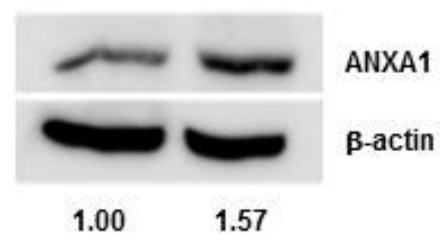

D

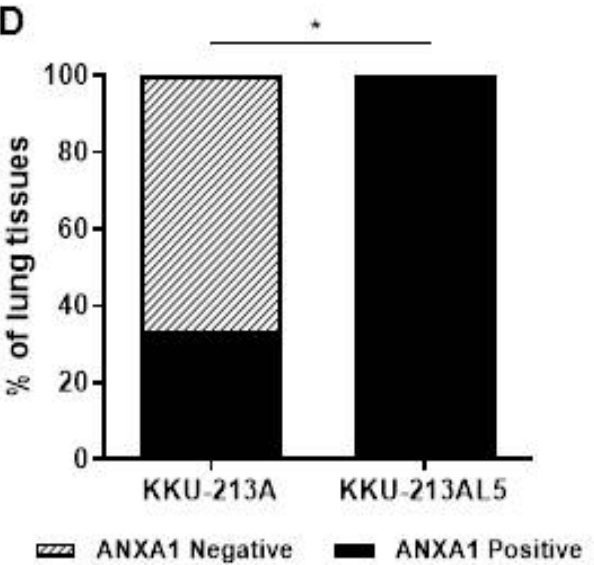

Figure 1. ANXA1 Expression in KKU-213A and KKU-213AL5 Cell Lines and Metastases of These in Mouse Lungs. Comparison of ANXA1 expression at (A) the mRNA level; and (B) protein level in KKU-213A and highly metastatic KKU-213AL5 cell lines. The expression of ANXA1 in KKU-213A was normalized with $\beta$-actin and set $=1$. (C) The expression of ANXA1 and CK19 in lung metastasized tissues from mice injected with KKU-213A and KKU-213AL5 was determined using IHC (magnification 40x). (D) The percentages of cells staining positive for ANXA1 in lung tissues from mice injected with KKU-213A or KKU-213AL5 CCA cells ( $\mathrm{n}=6$ each group). $* \mathrm{P}<0.05$ 
in the scramble control (Figure 2A). Cell proliferation was examined using the MTT assay. Viable cells cultured for 1, 2 and 3 days were compared between scramble control and si-ANXA1-treated KKU-213AL5 cells. As shown in Figure $2 \mathrm{~B}$, the proliferation of si-ANXA1 treated cells was significantly lower than the scramble control on days 1 to 3 .

The involvement of ANXA1 with colony formation was next investigated using a clonogenic assay. Cells were allowed to form colonies for 7 days and the number of colonies was counted. KKU-213AL5 cells treated with si-ANXA1 formed significantly fewer colonies than did scramble-control cells $(\mathrm{P}<0.001)$ as shown in Figure $2 \mathrm{C}$. The number of colonies in the scramble-control cells was $162.0 \pm 1.4$, whereas si-ANXA1-treated cells formed $100.8 \pm 7.0$ colonies.

In addition, migration and invasion abilities of si-ANXA1-treated KKU-213AL5 cells were also investigated using a Boyden chamber assay. Knockdown of ANXA1 expression dramatically reduced the number of migrating cells by $3 / 4$ and decreased the number of invading cells by half when compared with the scramble controls (Figure 2D-E).
A

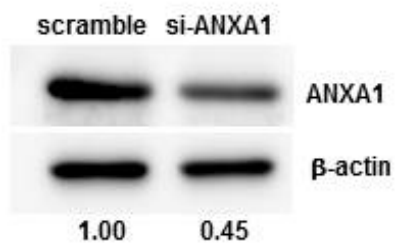

B

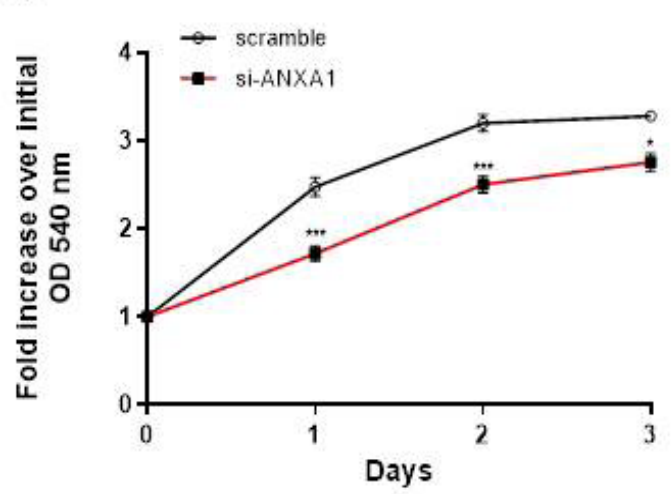

D
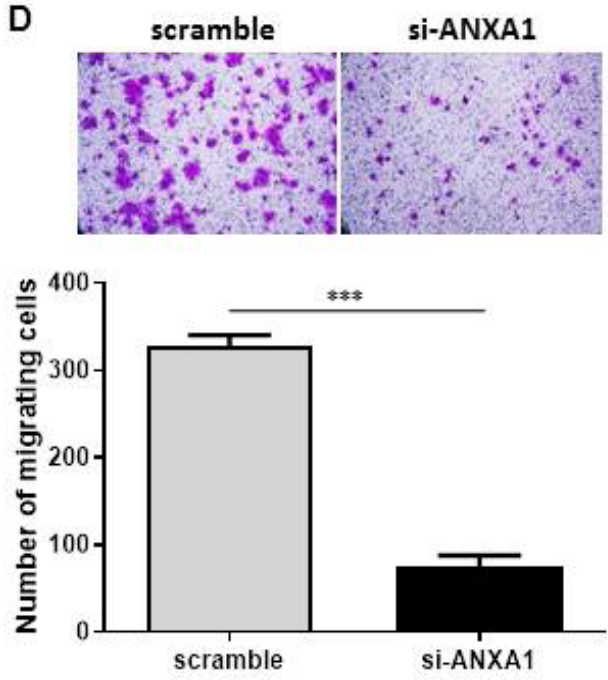

C
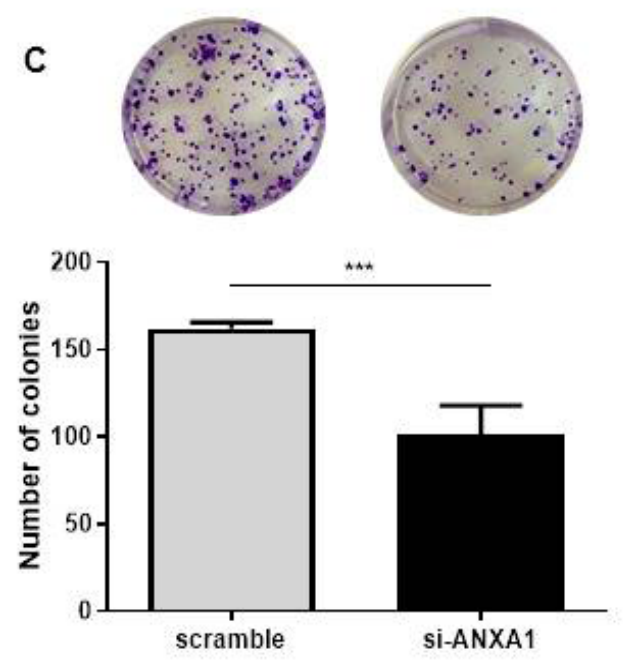

E
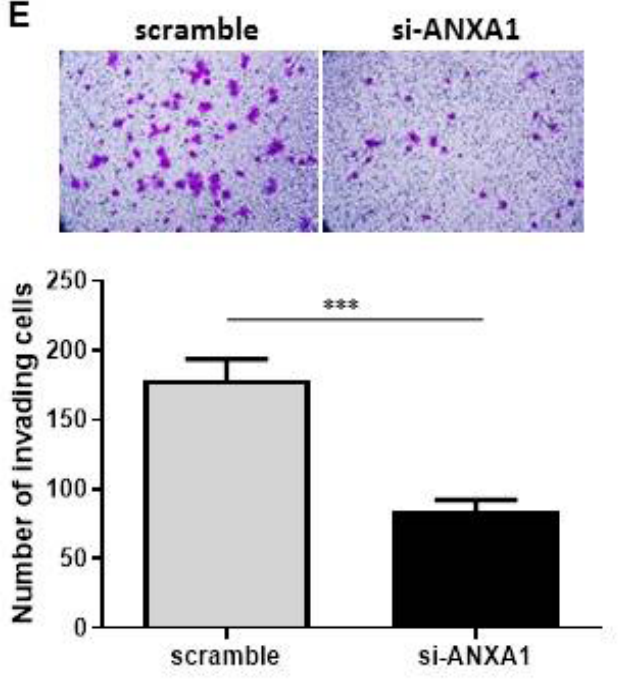

Figure 2. Suppression of ANXA1 Reduced Cell Proliferation, Colony Formation, Cell Migration and Invasion of KKU-213AL5 Cells. (A) Expression of ANXA1 protein was depleted by siRNA (si-ANXA1). (B) The rate of cell proliferation was determined daily for 3 days using the MTT assay. Relative proliferation was normalized to day 0 and compared between scramble control cells and si-ANXA1-treated cells. Data represent mean \pm SD of three replicates from one of two independent experiments. (C) Silencing of ANXA1 reduced colony formation in the KKU213AL5 cell line. Crystal violet-stained colonies of KKU-213AL5 compared between the scramble control and siANXA1-treated cells. Data are presented as the mean \pm SD of numbers of colonies in three replicates from one of two independent experiments. ${ }^{*} \mathrm{P}<0.05, * * * \mathrm{P}<0.001$. The numbers of migrating cells (D) and invading cells (E) of KKU-213AL5 compared between scramble control and si-ANXA1-treated cells. Data are presented as the mean \pm SD of numbers of migrating/ invading cells in three replicates from one of three independent experiments. $* * * \mathrm{P}<0.001$. 
High ANXA1 expression was associated with tumor staging and lymph-node metastasis in human CCA tissues

To verify the clinical importance of ANXA1 expression in CCA patients, IHC of ANXA1 protein was performed in 44 human CCA tissues of the mass-forming type. As shown in Figure 3A, there was little or no staining of ANXA1 protein in bile-duct epithelia of non-tumor counterparts. More intense staining was observed in CCA tissues. Comparisons of ANXA1 expression levels in 44 individual CCA patients between normal bile duct epithelium and CCA (Figure 3B). ANXA1 protein was undetectable in 11 of 44 cases $(25 \%)$ of bile-duct epithelia in non-tumor counterparts and expression was mostly low in the remaining cases. The mean IHC index for ANXA1 in CCA tissues $(6.89 \pm 2.73)$ was significantly higher than that for normal bile duct $(2 \pm 2.08)(\mathrm{P}<0.001)$, as presented in Figure 3C.

The associations between ANXA1 expression levels and clinicopathological features including age, sex, tumor staging, tumor size and lymph node metastasis were analyzed. As shown in Table 1, the median ANXA1-IHC index value $(=6)$ was used to divide CCA tissues into two categories: low $(\leq 6)$ versus high $(>6)$ levels of ANXA1 expression. There was no significant association

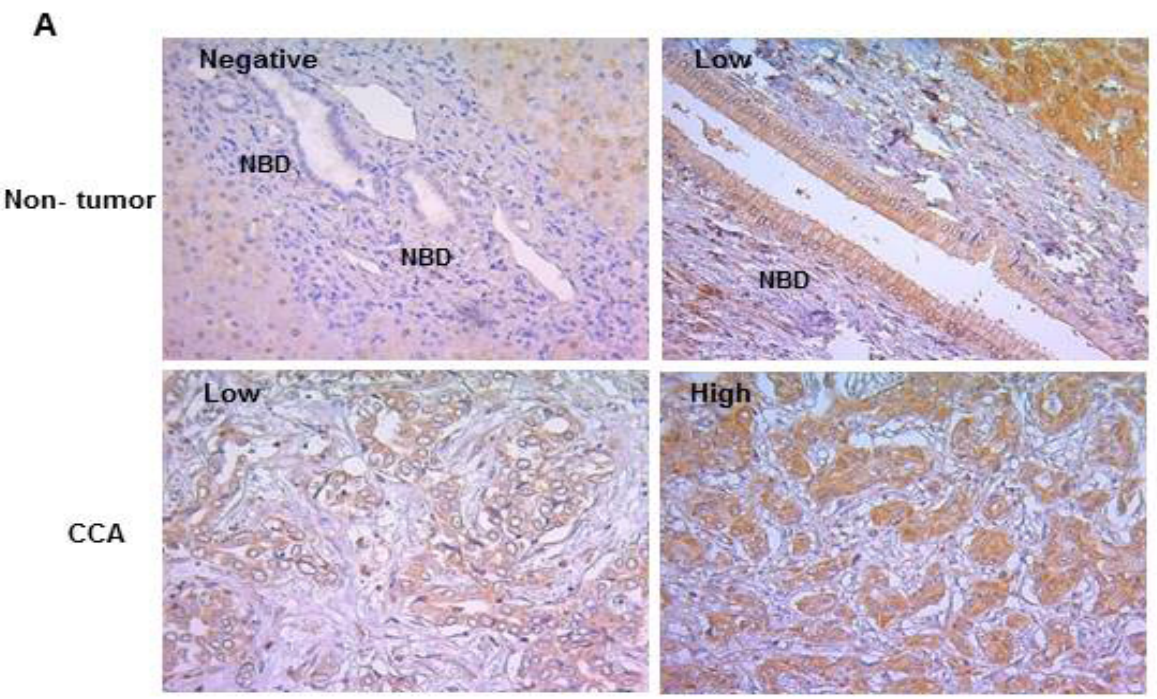

B

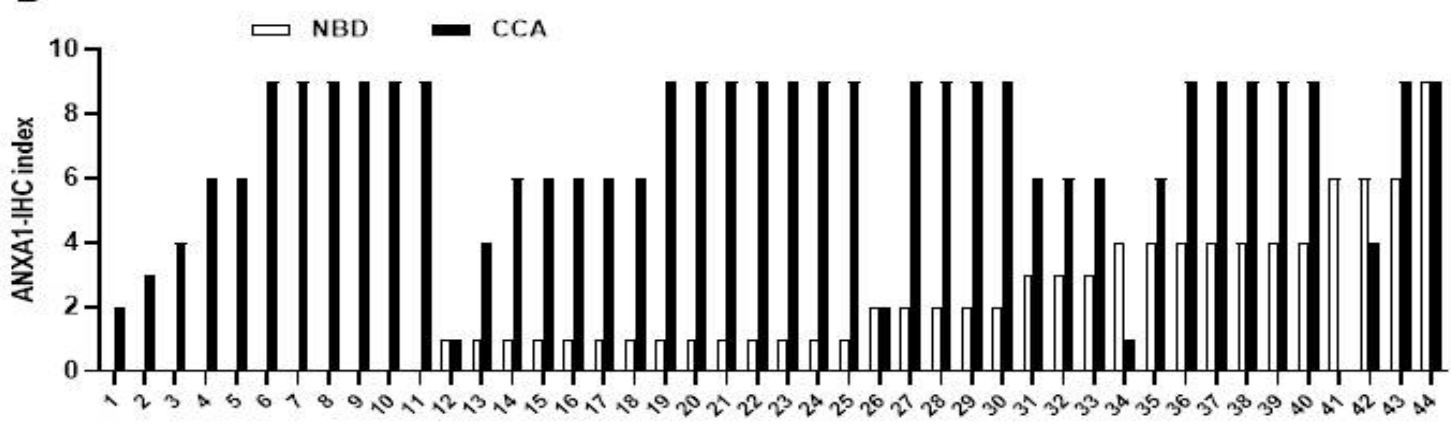

C

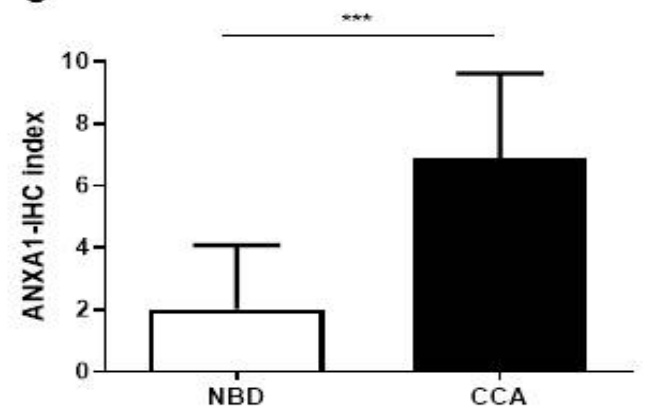

D

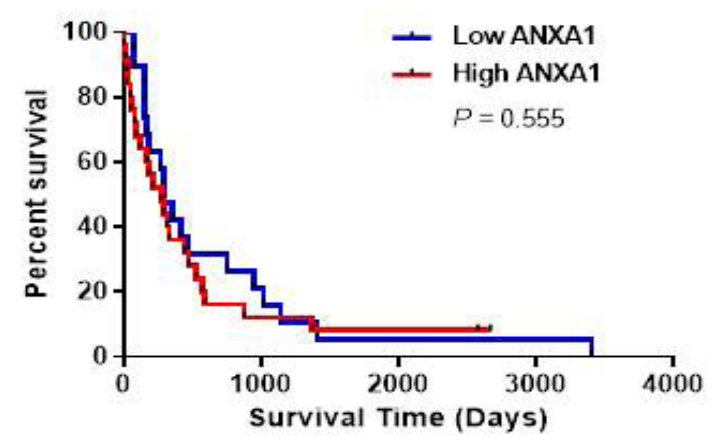

Figure 3. Immunohistochemistry of ANXA1 in Human CCA Tissues. (A) Negative or low expression of ANXA1 is apparent in non-tumor tissues. CCA tissues with low ANXA1 expression (IHC index $\leq 6$ ) and high ANXA1 expression (IHC index >6). NBD = normal bile duct, magnification 200x. (B) ANXA1 protein expression levels in each of 44 CCA patients. The IHC-index is shown of ANXA1 in normal bile duct (NBD) and CCA tissues from individual CCA patients. (C) Comparison of IHC indices of ANXA1 in NBD and CCA tissues. $* * * P<0.001$. (D) Kaplan-Meier survival plot between low and high ANXA1 expressing CCA patients. 
Table 1. Univariate Analysis of Low and High ANXA1 Expression in 44 CCA Patients

\begin{tabular}{|c|c|c|c|c|}
\hline \multirow[t]{2}{*}{ Variable } & \multirow[t]{2}{*}{$\mathrm{n}$} & \multicolumn{2}{|c|}{ ANXA1 expression } & \multirow[t]{2}{*}{$\overline{P \text { value }}$} \\
\hline & & Low $(\leq 6)$ & $\operatorname{High}(>6)$ & \\
\hline \multicolumn{5}{|l|}{ Age (years) } \\
\hline$\leq 56$ & 28 & 14 & 14 & 0.227 \\
\hline$>56$ & 16 & 5 & 11 & \\
\hline \multicolumn{5}{|l|}{ Sex } \\
\hline Female & 22 & 10 & 12 & $>0.999$ \\
\hline Male & 22 & 10 & 12 & \\
\hline \multicolumn{5}{|c|}{ Tumor staging } \\
\hline III & 10 & 7 & 3 & 0.027 \\
\hline IVA & 21 & 10 & 11 & \\
\hline IVB & 13 & 2 & 11 & \\
\hline \multicolumn{5}{|c|}{ Tumor size* $(\mathrm{cm})$} \\
\hline$\leq 5$ & 17 & 11 & 6 & 0.042 \\
\hline$>5 \mathrm{~cm}$ & 27 & 9 & 18 & \\
\hline \multicolumn{5}{|c|}{ Lymph node metastasis } \\
\hline No & 24 & 15 & 9 & 0.017 \\
\hline Yes & 20 & 5 & 15 & \\
\hline
\end{tabular}

*Tumor size based on longest diameter

of ANXA1 expression with age or sex. However, high ANXA1 expression was significantly associated with tumor staging $(\mathrm{P}=0.027)$, tumor size $(\mathrm{P}=0.042)$ and lymph-node metastasis $(\mathrm{P}=0.017)$. The median survival time of these two groups was not significant different (low ANXA1, 302 days versus high ANXA1, 275 days) as shown in Figure 3D.

\section{Discussion}

ANXA1 is a calcium-binding protein involved in diverse cellular functions, such as inflammation, proliferation and apoptosis. The contribution of ANXA1 to tumorigenesis and metastasis is widely recognized (de Graauw et al., 2010; Kang et al., 2012; Han et al., 2017b). However, the exact mechanisms by which ANXA1 exerts its effects in CCA progression are still not clearly understood. In our study, ANXA1 was overexpressed in the highly metastatic KKU-213AL5 cell line, both at the mRNA and the protein level. Association of ANXA1 expression with metastatic properties of KKU-213AL5 was also confirmed in the xenograft tissues. ANXA1 was overexpressed in primary tumor sections and metastasized lung sections of highly metastatic KKU-213AL5 cell line when compared with the parental KKU-213A cell line. Similar observations have been reported in rat and human mammary-adenocarcinoma tissues, showing ANXA1 was highly expressed in lung metastatic tumors compared with parental cells (Pencil and Toth, 1998). An association between ANXA1 expression and metastatic activity was also shown in highly invasive mouse breast-cancer cells (Okano et al., 2015).

Our study demonstrated that suppression of ANXA1 using specific siRNA significantly reduced cell proliferation, colony formation, migration and invasion in KKU-213AL5 cells. The findings were consistent with multiple types of cancers (Babbin et al., 2006; de Graauw et al., 2010; Khau et al., 2011; Belvedere et al., 2014; Zhao et al., 2021), strongly suggesting ANXA1 contributed to metastasis of cancers. Knockdown of ANXA1 diminished the mRNA expression of transforming growth factor- $\beta$ (TGF- $\beta$ ) and 2 types of matrix metalloproteinase (MMP2 and MMP9) in CCA cell lines (Hongsrichan et al., 2013). ANXA1 may regulate TGF- $\beta$ signaling and induce epithelial-mesenchymal transition (EMT) (de Graauw et al., 2010) leading to up-regulation of MMP2 and MMP9 (Wiercinska et al., 2011), which in turn degrades extracellular matrix (ECM), promoting cancer progression and metastasis. This supports the role of ANXA1 in CCA metastasis. In addition, overexpression of ANXA1 enhances the invasion and metastasis of esophageal squamous-cell carcinoma by inducing the Snail/E-cadherin pathway (Han et al., 2017b). To gain a better understanding of the molecular mechanisms underlying ANXA1-associated metastasis of CCA, it will be interesting to study expression of other EMT-related proteins in ANXA1-knockdown CCA cell lines . Ectopic overexpression of ANXA1 may be another tool for investigating the migratory/invasive properties of low ANXA1-expressing cells. Moreover, the role of ANXA1 in the progression of CCA should be further investigated in a preclinical experiment.

High expression of ANXA1 in CCA tissues was associated with advanced tumor stage and presence of lymph-node metastasis. This is consistent with other studies of metastasis in various cancers (Kang et al., 2002; Wang et al., 2006a; Wang et al., 2006b; de Graauw et al., 2010; He et al., 2010; Bist et al., 2011; Sato et al., 2011; Biaoxue et al., 2012; Kang et al., 2012; Gastardelo et al., 2014; Okano et al., 2015; Han et al., 2017a; Han et al., 2017b; Pessolano et al., 2018). This body of research strongly supports the clinical significance of ANXA1 as a metastatic marker of CCA.

In conclusion, we have demonstrated that high expression levels of ANXA1 are associated with advanced tumor stage, tumor size and lymph-node metastasis in CCA patients. These findings strongly indicate that ANXA1 is a potential prognostic marker and may be used to screen CCA patients who are at risk of metastasis. Suppression of ANXA1 impeded proliferation, invasion and metastasis of a highly metastatic CCA cell line. Our research may prove useful in targeting ANXA1 for inhibition of metastasis in CCA patients. Further studies of the underlying mechanism are required to warrant our findings.

\section{Author Contribution Statement}

Conceptualization, KS; Formal analysis, KUK, $\mathrm{SuO}, \mathrm{KV}, \mathrm{SO}, \mathrm{SW}$ and KS; Investigation, KUK and KS; Supervision, SO and SW; Writing - original draft, KUK and KS; Writing - review \& editing, KUK, $\mathrm{SuO}, \mathrm{KV}$, SO, SW and KS. 


\section{Acknowledgements}

We thank Prof. David Blair for editing this manuscript via Publication Clinic KKU, Thailand.

\section{Funding statement}

This study received funding support from the National Science, Research and Innovation Fund, Khon Kaen University (to KS).

\section{Conflict of interest statement}

The authors declare no conflict of interest.

\section{References}

Babbin BA, Lee WY, Parkos CA, et al (2006). Annexin I regulates SKCO-15 cell invasion by signaling through formyl peptide receptors. J Biol Chem, 281, 19588-99.

Belvedere R, Bizzarro V, Popolo A, et al (2014). Role of intracellular and extracellular annexin A1 in migration and invasion of human pancreatic carcinoma cells. $B M C$ Cancer, 14, 961.

Biaoxue R, Xiling J, Shuanying Y, et al (2012). Upregulation of Hsp90-beta and annexin A1 correlates with poor survival and lymphatic metastasis in lung cancer patients. J Exp Clin Cancer Res, 31, 70.

Bist P, Leow SC, Phua QH, et al (2011). Annexin-1 interacts with NEMO and RIP1 to constitutively activate IKK complex and NF-kappaB: implication in breast cancer metastasis. Oncogene, 30, 3174-85.

Bradford MM (1976). A rapid and sensitive method for the quantitation of microgram quantities of protein utilizing the principle of protein-dye binding. Anal Biochem, 72, 248-54.

Butthongkomvong K, Sirachainan E, Jhankumpha S, et al (2013). Treatment outcome of palliative chemotherapy in inoperable cholangiocarcinoma in Thailand. Asian Pac J Cancer Prev, 14, 3565-8.

Caron D, Maaroufi H, Michaud S, et al (2013). Annexin A1 is regulated by domains cross-talk through post-translational phosphorylation and SUMOYlation. Cellular Signalling, 25, 1962-9.

de Graauw M, van Miltenburg MH, Schmidt MK, et al (2010). Annexin A1 regulates TGF-beta signaling and promotes metastasis formation of basal-like breast cancer cells. Proc Natl Acad Sci U S A, 107, 6340-5.

Franken NA, Rodermond HM, Stap J, et al (2006). Clonogenic assay of cells in vitro. Nat Protoc, 1, 2315-9.

Gastardelo TS, Cunha BR, Raposo LS, et al (2014). Inflammation and cancer: role of annexin A1 and FPR2/ALX in proliferation and metastasis in human laryngeal squamous cell carcinoma. PLoS One, 9, e111317.

Han F, Shrestha S, Huang H, et al (2017a). Expression of annexin II in gastric carcinoma and its role in gastric cancer metastasis. World J Gastroenterol, 23, 7009-15.

Han G, Lu K, Huang J, et al (2017b). Effect of Annexin A1 gene on the proliferation and invasion of esophageal squamous cell carcinoma cells and its regulatory mechanisms. Int $J$ Mol Med, 39, 357-63.

He ZY, Wen H, Shi CB, et al (2010). Up-regulation of hnRNP $\mathrm{A} 1$, Ezrin, tubulin beta-2C and Annexin A1 in sentinel lymph nodes of colorectal cancer. World J Gastroenterol, 16, 4670-6.

Hongsrichan N, Rucksaken R, Chamgramol Y, et al (2013). Annexin A1: A new immunohistological marker of cholangiocarcinoma. World J Gastroenterol, 19, 2456-65.

Kang H, Ko J, Jang SW (2012). The role of annexin A1 in expression of matrix metalloproteinase-9 and invasion of breast cancer cells. Biochem Biophys Res Commun, 423, 188-94.

Kang JS, Calvo BF, Maygarden SJ, et al (2002). Dysregulation of annexin I protein expression in high-grade prostatic intraepithelial neoplasia and prostate cancer. Clin Cancer Res, 8, 117-23.

Khau T, Langenbach SY, Schuliga M, et al (2011). Annexin-1 signals mitogen-stimulated breast tumor cell proliferation by activation of the formyl peptide receptors (FPRs) 1 and 2. FASEB J, 25, 483-96.

Laemmli UK (1970). Cleavage of structural proteins during the assembly of the head of bacteriophage T4. Nature, 227, 680-5.

Lim LH, Pervaiz S (2007). Annexin 1: the new face of an old molecule. FASEB journal : official publication of the Federation of American Societies for Experimental Biology, 21, pp 968-75.

Luvira V, Nilprapha K, Bhudhisawasdi V, et al (2016). Cholangiocarcinoma patient outcome in northeastern Thailand: Single-Center Prospective Study. Asian Pac J Cancer Prev, 17, 401-6.

Okano M, Kumamoto K, Saito M, et al (2015). Upregulated Annexin A1 promotes cellular invasion in triple-negative breast cancer. Oncol Rep, 33, 1064-70.

Pencil SD, Toth M (1998). Elevated levels of annexin I protein in vitro and in vivo in rat and human mammary adenocarcinoma. Clin Exp Metastasis, 16, 113-21.

Pessolano E, Belvedere R, Bizzarro V, et al (2018). Annexin A1 may induce pancreatic cancer progression as a key player of extracellular vesicles effects as evidenced in the in vitro MIA PaCa-2 model system. Int J Mol Sci, 19.

Sato Y, Kumamoto K, Saito K, et al (2011). Up-regulated Annexin A1 expression in gastrointestinal cancer is associated with cancer invasion and lymph node metastasis. Exp Ther Med, 2, 239-43.

Uthaisar K, Vaeteewoottacharn K, Seubwai W, et al (2016). Establishment and characterization of a novel human cholangiocarcinoma cell line with high metastatic activity. Oncol Rep, 36, 1435-46.

Wang AG, Yoon SY, Oh JH, et al (2006a). Identification of intrahepatic cholangiocarcinoma related genes by comparison with normal liver tissues using expressed sequence tags. Biochem Biophys Res Commun, 345, 1022-32.

Wang KL, Wu TT, Resetkova E, et al (2006b). Expression of annexin A1 in esophageal and esophagogastric junction adenocarcinomas: association with poor outcome. Clinical cancer research : an official journal of the American Association for Cancer Research, 12, pp 4598-604.

Wiercinska E, Naber HP, Pardali E, et al (2011). The TGF-beta/ Smad pathway induces breast cancer cell invasion through the up-regulation of matrix metalloproteinase 2 and 9 in a spheroid invasion model system. Breast Cancer Res Treat, 128, 657-66.

Zeng X, Tao H (2015). Diagnostic and prognostic serum marker of cholangiocarcinoma (Review). Oncol Lett, 9, 3-8.

Zhao X, Ma W, Li X, et al (2021). ANXA1 enhances tumor proliferation and migration by regulating epithelialmesenchymal transition and IL-6/JAK2/STAT3 pathway in papillary thyroid carcinoma. J Cancer, 12, 1295-306.

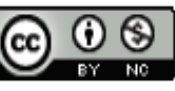

This work is licensed under a Creative Commons AttributionNon Commercial 4.0 International License. 